\title{
Pattern Recognition to Objectively Differentiate the Etiology of Cognitive Decline: Analysis of the Impact of Stroke and Alzheimer's Disease
}

\author{
Sean A.P. Clouston ${ }^{a}$ Lauren L. Richmond ${ }^{b}$ Stacey B. Scott ${ }^{b}$ \\ Christian C. Luhmann ${ }^{\text {b, d }}$ Ginny Natale ${ }^{c}$ Douglas Hanes ${ }^{c}$ Yun Zhang ${ }^{c}$ \\ Dylan M. Smith ${ }^{\mathrm{a}}$
}

aProgram in Public Health and Department of Family, Population, and Preventive Medicine, Renaissance School of Medicine at Stony Brook University, Stony Brook, NY, USA; 'b Department of Psychology, Stony Brook University, Stony Brook, NY, USA; 'Program in Public Health, Renaissance School of Medicine at Stony Brook University, Stony Brook, NY, USA; ${ }^{~ I n s t i t u t e ~ f o r ~ A d v a n c e d ~ C o m p u t a t i o n a l ~ S c i e n c e, ~ S t o n y ~ B r o o k ~ U n i v e r s i t y, ~ S t o n y ~ B r o o k, ~ N Y, ~ U S A ~}$

\section{Keywords \\ Neuroepidemiology · Cerebrovascular disease · Pattern recognition - Adaptive diagnostics}

\begin{abstract}
Background: Undetected Alzheimer's disease (AD) and stroke neuropathology is believed to account for a large proportion of decline in cognitive performance that is attributed to normal aging. This study examined the amount of variance in age-related cognitive change that is accounted for by $A D$ and stroke using a novel pattern recognition protocol. Method: Secondary analyses of data collected for the Health and Retirement Study $(N=17,579)$ were used to objectively characterize patterns of cognitive decline associated with $A D$ and stroke. The rate of decline in episodic memory and orientation was the outcome of interest, while algorithms indicative of $A D$ and stroke pathology were the predictors of interest. Results: The average age of the sample was $67.54 \pm$ 10.45 years at baseline, and they completed, on average, $14.20 \pm 3.56$ years of follow-up. After adjusting for demographics, AD and stroke accounted for approximately half of age-associated decline in cognition $(51.07-55.6 \%$ for orientation and episodic memory, respectively) and explained
\end{abstract}

karger@karger.com www.karger.com/ned

Karger $\stackrel{\text { ' }}{5}$

GOPEN ACCESS
(C) 2020 The Author(s)

Published by S. Karger AG, Basel

This is an Open Access article licensed under the Creative Commons Attribution-NonCommercial-4.0 International License (CC BY-NC) (http://www.karger.com/Services/OpenAccessLicense), applicable to the online version of the article only. Usage and distribution for commercial purposes requires written permission. variance attributed to random slopes in longitudinal multilevel models. Discussion: The results of this study suggested that approximately half of the cognitive decline usually attributed to normal aging are more characteristic of $A D$ and stroke.

(C) 2020 The Author(s)

Published by S. Karger AG, Basel

\section{Introduction}

Alzheimer's disease and related dementias are extremely common and are estimated to affect 14 million people in the United States [1], and was recorded as the underlying cause of death in $\geq 110,000$ cases in 2018 [2]. More than $99 \%$ of all dementias fall into two main types: Alzheimer's disease (AD), which accounts for approximately $60-80 \%$ of all cases, and vascular dementia, primarily associated with major stroke in addition to other cerebrovascular causes and risk factors. Both AD and vascular dementia are known to impair cognitive performance [3]. However, dementias due to AD and cerebrovascular diseases including ischemic events (i.e., major and silent "stroke") are often characterized by substantial losses in cognitive functioning that can take years to ac- 


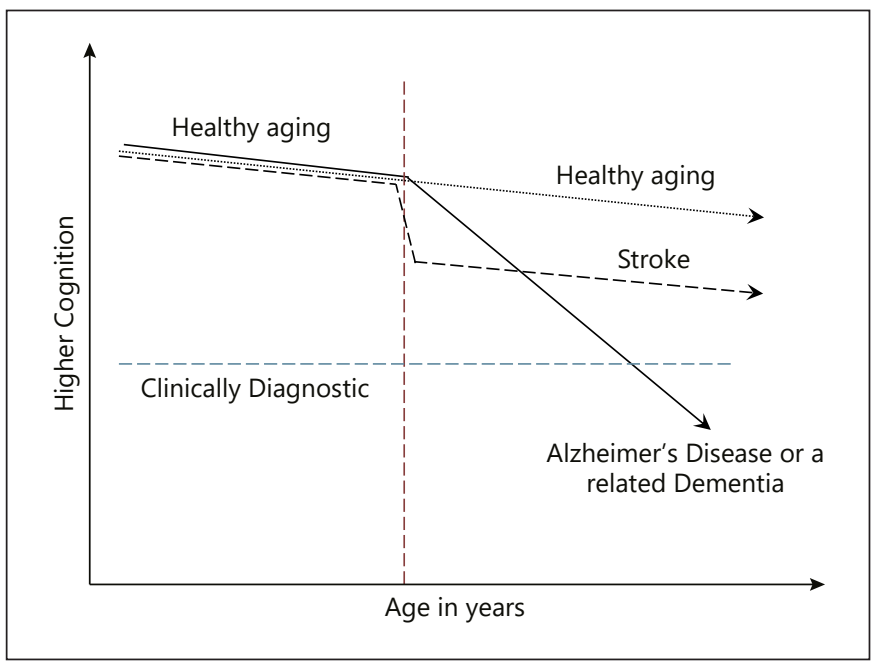

Fig. 1. Differential pathological characterization based on aging pattern in episodic memory: vascular decline occurs quickly but stabilizes while Alzheimer's-related decline represents slow but progressive declines in cognitive function with time.

cumulate in order to cause clinical levels of cognitive impairment necessary for diagnosis of dementia.

Recent work has suggested that approximately half (41-52\%) of age-related cognitive decline observed in older adult populations may be attributable to the presence of age-related pathologies including $\mathrm{AD}$ and stroke $[4,5]$. However, efforts thus far have been limited because disease diagnosis, identification of causes, and rates of progression rely heavily on subjective reports from individuals and/or informants to characterize disease progress. Yet, clinical diagnoses for $\mathrm{AD}$ and stroke have long been differentiated based on the rate at which cognitive declines occur [6]. However, to date these diagnoses have relied almost exclusively on subjective individual or informant reports about rapidity of symptom onset to help differential diagnosis of $\mathrm{AD}$ and stroke [7]. However, whereas "normal aging" in cognitive performance is conceptualized as occurring at a linear rate throughout adulthood [8], cognitive decline evident in $\mathrm{AD}$ (as represented graphically in Fig. 1) is characterized by decades [9] of progressive accelerated losses in cognitive functioning when compared to normal aging [10], and cognitive decline in stroke is often clinically characterized by a rapid loss in functioning which causes losses over a very short period of time before eventually stabilizing [11].

This study relies on recent advances in pattern recognition algorithms to clinically differentiate normal aging from $\mathrm{AD}[12]$ and ischemic events (i.e., major and silent "stroke") [13]. The goal of this study was to determine the extent to which AD and stroke-like events identified using pattern recognition algorithms helped explain associations between aging and cognitive decline in episodic memory and orientation in a national sample of older US residents.

\section{Methods}

We utilized data from the Health and Retirement Study (HRS), a large representative sample of older adults in the USA, which collects cognitive data biennially starting in 1992 (response rate $81.6 \%)$. The HRS is open to enrollment at subsequent waves, and data are publicly available online (http://hrsonline.isr.umich.edu) [14]. Data from waves 3-12 were used in this analysis due to different parameters for tasks of interest in waves 1 and 2 compared to those used in waves 3 and beyond. Due to the intense analytic requirements for differential diagnostic routines, respondents without at least 4 valid observations were excluded. The analytic sample included 17,579 respondents who were followed up 7.9 times, on average, over the course of $14.20(\mathrm{SD}=3.56)$ years.

\section{Measures}

Episodic memory is a critical measure of cognitive functioning that is sensitive to both cognitive aging, $\mathrm{AD}$ [15], and, in its acute phase, stroke [16]. To measure episodic memory, respondents were first provided with a list of 10 unrelated words and asked to immediately recall as many words as they could remember from the initial list in any order that they wished. Participants reported each recalled item aloud to the interviewer, and each correctly recalled word was worth one point. After completing a distractor task that lasted 10-15 min, respondents were again asked to correctly recall as many words from the initial list as possible. Recalled items were reported aloud to the interviewer, with each correctly recalled item scoring a single point irrespective of the order in which the items were recalled. The verbal episodic memory index included the summation of both immediate and delayed tests (possible scores ranging from 0 to 20 ). The resulting index was normally distributed (mean $=10.83, \mathrm{SD}=3.44$, skew $=-0.05$, kurtosis $=2.94)$.

Orientation is a common measure of dementia risk including both Alzheimer's disease and vascular dementia. In the HRS, orientation was measured by testing participants on knowledge of common everyday facts, such as the day of the week or the current president's name, and the capacity to complete simple financial numeracy tasks. Confusion in these domains is believed to indicate disorientation in everyday life consistent with dementia, including Alzheimer's disease and vascular dementia. Measures also included determining similarities between common items, identification of pictures of common items, and being able to correctly count and subtract numbers in reverse. A factor analysis clarified that these measures formed a single-factor solution $(\xi=0.81$; weights were 0.32 [backward counting], 0.50 [subtract sevens], and 0.48 [orientation]). In the HRS, orientation (with possible scores ranging from 0 to 15) is measured among individuals aged 65 and older using 7 questions including the day of the week, the date including day, month, and year (e.g., "April 4, 2018”); numeracy is measured using the ability to subtract seven from a random number and the 
Table 1. Descriptive characteristics of eligible participants, and separately as characterized by the presence of Alzheimer's disease and/or stroke-like cognitive pathology, $\operatorname{HRS}(N=17,579)$

\begin{tabular}{|c|c|c|c|c|c|c|c|c|c|c|}
\hline \multirow[t]{2}{*}{ Characteristic } & \multicolumn{2}{|c|}{ Whole sample } & \multicolumn{2}{|c|}{$\begin{array}{l}\text { Normal } \\
(n=6,601\end{array}$} & \multicolumn{2}{|c|}{$\begin{array}{l}\text { Alzheimer's disease } \\
\text { only }(n=1,799 ; \\
10.23 \%)\end{array}$} & \multicolumn{2}{|c|}{$\begin{array}{l}\text { Stroke only } \\
(n=4,661 ; \\
26.51 \%)\end{array}$} & \multicolumn{2}{|c|}{$\begin{array}{l}\text { Mixed or both } \\
(n=4,518 \\
25.70 \%)\end{array}$} \\
\hline & mean & $\mathrm{SD}$ & mean & $\mathrm{SD}$ & mean & SD & mean & $\mathrm{SD}$ & mean & SD \\
\hline Episodic memory in words & 9.99 & 3.57 & 10.41 & 3.34 & 10.55 & 3.22 & $9.95^{\dagger}$ & 3.58 & $9.21^{\dagger}$ & 3.88 \\
\hline Orientation & 12.67 & 2.48 & 12.86 & 2.31 & $12.66^{\dagger}$ & 2.46 & 12.91 & 2.21 & $12.39^{\dagger}$ & 2.73 \\
\hline \multirow[t]{2}{*}{ Age, years } & 60.27 & 9.51 & 57.67 & 8.34 & $60.18^{\dagger}$ & 9.74 & 57.62 & 6.99 & $65.21^{\dagger}$ & 9.78 \\
\hline & \multicolumn{2}{|r|}{$\%$} & \multicolumn{2}{|r|}{$\%$} & \multicolumn{2}{|r|}{$\%$} & \multicolumn{2}{|r|}{$\%$} & \multicolumn{2}{|r|}{$\%$} \\
\hline \multicolumn{11}{|l|}{ Race/ethnicity } \\
\hline White & \multicolumn{2}{|r|}{75.84} & \multicolumn{2}{|r|}{75.03} & \multicolumn{2}{|r|}{73.51} & \multicolumn{2}{|r|}{75.46} & \multicolumn{2}{|r|}{79.09} \\
\hline Black & \multicolumn{2}{|r|}{13.50} & \multicolumn{2}{|r|}{14.07} & \multicolumn{2}{|r|}{14.63} & \multicolumn{2}{|r|}{13.32} & \multicolumn{2}{|r|}{12.23} \\
\hline Other & \multicolumn{2}{|r|}{2.11} & \multicolumn{2}{|r|}{2.06} & \multicolumn{2}{|r|}{2.44} & \multicolumn{2}{|r|}{2.13} & \multicolumn{2}{|r|}{1.88} \\
\hline Hispanic & \multicolumn{2}{|r|}{8.56} & \multicolumn{2}{|r|}{8.84} & \multicolumn{2}{|r|}{9.43} & \multicolumn{2}{|r|}{9.10} & \multicolumn{2}{|r|}{6.80} \\
\hline Male sex & \multicolumn{2}{|r|}{38.25} & \multicolumn{2}{|r|}{37.74} & \multicolumn{2}{|r|}{39.96} & \multicolumn{2}{|r|}{39.09} & \multicolumn{2}{|r|}{36.38} \\
\hline
\end{tabular}

Diagnostic categories were determined using pattern recognition protocols. $p$ values for continuous variables were derived from nonparametric trend tests showing whether there were any differences between the diagnostic types. SD, standard deviation; HRS, Health and Retirement Study. ${ }^{\dagger} p<1 \mathrm{E}-06$.

ability to count backward from another number; object naming for one man-made object and another natural object; and the names of the current president and vice president of the USA.

\section{Alzheimer's and Stroke Pathological Declines}

Under normal aging, which is often defined as the decline that is evident among individuals who are not exhibiting signs of a cognitively impairing disease [8], cognitive decline occurs linearly at a steady rate that may differ between individuals. These methods have been described in depth elsewhere [12,17]; briefly, the pattern recognition model sorts through the longitudinal data seeking a pattern defined a priori to match clinical expectations of Alzheimer's disease, stroke, or normal aging. As such, a linear rate of decline was used as the null assumption; whereas a pattern of piecewise-linear accelerated decline was identified for Alzheimer's disease, a pattern of significant stepwise decline was identified for stroke (following Fig. 1). To objectively detect Alzheimer's-like and/or stroke-like patterns of decline, a pattern recognition protocol was applied to individual-level trajectories of episodic memory to determine which pattern maximized the log-likelihood; model fit statistics were retained. These trajectories were used both to characterize individuals as having either $\mathrm{AD}$ or stroke and to identify the timing of onset for $\mathrm{AD}$ and/or stroke-like disease or "cognitive pathology". Akaike's information criterion was used to compare linear models of healthy aging to pathological models for each individual, and then to differentiate between stroke and AD-related cognitive pathology. In cases where both $\mathrm{AD}$ and stroke pathologies were identified, a mixed (both were identified and fit improved with both) or indeterminate (both were identified but neither disease was primary) category was also recorded for descriptive purposes. For the purposes of contextualizing these results, rates of decline were compared to each other and were compared with the average level of decline necessary to cause clinical diagnosis of amnestic mild cognitive impairment, as characterized by a loss of 1.5 standard deviations in episodic memory. Subgroup analyses examined the mixed category to examine rapidity of rates of decline in this group. Models incorporated the respondent's age in years, sex, and race/ethnicity.

\section{Statistical Analyses}

Means, standard deviations, and percentages were used to describe the sample. Prevalence of disease outcomes in the sample was estimated using percentages and 95\% confidence intervals (95\% CI). For descriptive purposes, age-adjusted incidence rates were also reported alongside accompanying 95\% CI.

Longitudinal multilevel modeling is a common method used to mark the rate of cognitive decline [18]. In longitudinal multilevel models, random intercepts were utilized to account for time-invariant individual differences in cognitive performance at baseline, while random slopes were utilized to account for heteroskedasticity in longitudinal trajectories [19]. An unstructured covariance matrix was utilized to account for potential associations between individual cognitive performance at baseline and rate of change over time; when negative, this association is attributable to regression to the mean [20]. Predicted results were used to provide model-estimated trajectories. The modeling strategy tested the assumption that age was associated with cognitive decline using age in years specified linearly; random intercepts and slopes were included in all modeling efforts. The beta coefficient for age in years was considered to capture the aging process. Multivariable adjusted models were then used to explain aging. These models included adjustments for race/ethnicity and sex as a base model, while a second model further examined the impact of adjusting for $\mathrm{AD}$ and stroke. The proposed method uses a nonlinear pattern recognition software to differentiate causes of cognitive decline. Since prior work has identified quadratic change in aging as a poten- 
Table 2. Coefficients and standard deviations examining associations between age, stroke, and Alzheimer's disease in explaining longitudinal declines in episodic memory

\begin{tabular}{|c|c|c|c|c|}
\hline \multirow[t]{2}{*}{ Fixed effect } & \multicolumn{2}{|c|}{ Model 1* } & \multicolumn{2}{|c|}{ Model 2* } \\
\hline & mean & SE & mean & SE \\
\hline Male sex & -0.947 & 0.035 & -1.098 & 0.037 \\
\hline Age, years & -0.152 & 0.001 & -0.068 & 0.001 \\
\hline \multicolumn{5}{|l|}{ Race/ethnicity } \\
\hline White & \multicolumn{2}{|c|}{ Reference } & \multicolumn{2}{|c|}{ Reference } \\
\hline Black & -1.789 & 0.051 & -1.681 & 0.053 \\
\hline Other & -1.238 & 0.119 & -1.007 & 0.125 \\
\hline Hispanic & -1.862 & 0.061 & -1.711 & 0.064 \\
\hline Stroke & & & -1.635 & 0.023 \\
\hline Alzheimer's disease & & & -0.896 & 0.006 \\
\hline Constant & 13.383 & 0.033 & 12.560 & 0.032 \\
\hline \multirow[t]{2}{*}{ Random effect } & \multicolumn{2}{|c|}{ Model 1* } & \multicolumn{2}{|c|}{ Model 2* } \\
\hline & ES & SE & ES & SE \\
\hline Slopes, SD & 0.127 & 0.002 & 0.000 & 0.000 \\
\hline Intercepts, SD & 2.342 & 0.018 & 2.262 & 0.014 \\
\hline Corr $(S, I)$ & -0.437 & 0.011 & 0.000 & 0.000 \\
\hline
\end{tabular}

SE, standard error; ES, estimate size. ${ }^{*}$ All nonzero associations shown were statistically significant at $p<1 \mathrm{E}-06$ level. Analyses additionally adjusted for practice effects observed between the first and following waves.

tially important component of the aging process, sensitivity analyses considered the role of a quadratic acceleration curve. All analyses were completed using Stata 15/SE (StataCorp).

\section{Results}

The sample was in their 60 s on average and made up predominantly of females (Table 1). Bivariate results revealed that individuals with any indication of pathological cognitive decline were older than those without, and that those with performance patterns consistent with $\mathrm{AD}$ or indeterminate patterns of decline were older than those with patterns consistent with stroke. Half of the respondents lacked patterns indicative of either $\mathrm{AD}$ or stroke. Not shown in Table 1, age-standardized rates were adjusted incidence rate $=34.57[29.38-39.77] / 1,000$ person-years for $\mathrm{AD}$ and 22.73 [17.69-27.76]/1,000 personyears for stroke.

The unadjusted rate of decline in episodic memory was $-0.146(95 \% \mathrm{CI}=[-0.144,-0.149])$ per year of decline after adjusting for random intercepts and slopes and

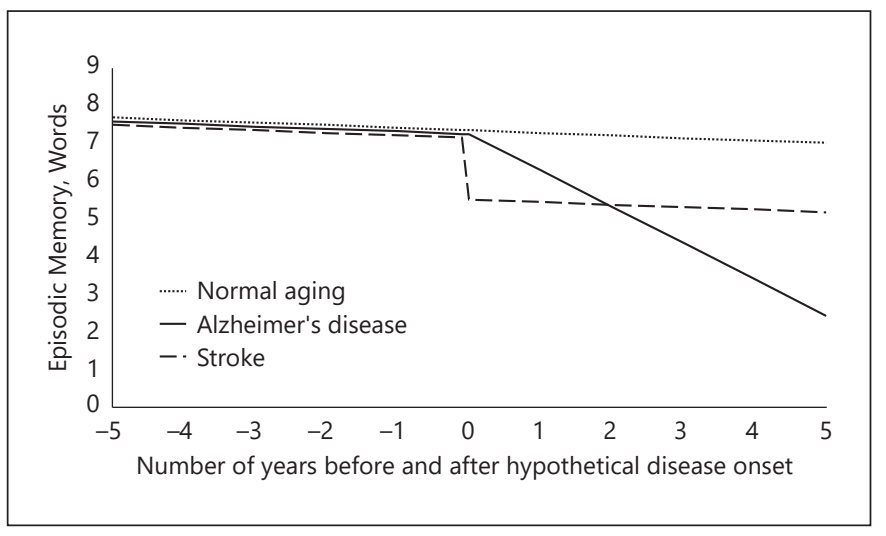

Fig. 2. Predicted episodic memory trajectories for normal aging, stroke, and Alzheimer's disease detected using pattern recognition methodology, HRS. HRS, Health and Retirement Study. Note: Results were generated using posterior predictions from Table 2, model 2.

their association (estimated: $-0.434[-0.412,-0.456]$ ). After adjusting for cross-sectional differences in sex and race and for practice effects (Table 2; model 1 ), the rate of age-related decline increased to $-0.152(95 \% \mathrm{CI}=[-0.150$, $-0.155]$ ) words per year of age. Finally, adjusting for both stroke and Alzheimer's patterns (Table 2; model 2), associations decreased by $55.6 \%[53.29,57.76]$ to -0.068 $[-0.065,-0.070]$ words/year. Additionally, integration of stroke and Alzheimer's patterns explained variance attributed to random slopes and negative associations found between random intercepts and slopes. Analyses suggested that while the effects of strokes are immediately evident (as represented in Fig. 2), the rate of progressive decline indicative of an $\mathrm{AD}$ pattern overtook the effects of a single stroke after 1.82 [1.80-1.85] years. The $\mathrm{AD}$ pattern was associated with reductions in episodic memory of 1.5 SDs over the course of 5.98 years. In contrast, the rate of decline in normal cognitive aging shown here $(B=-0.068)$ would take an equivalent of 13.17 years of normal aging to equate to the size of declines seen in only a single year of AD (0.896 words), and would cause only a decrement of 1.5 SDs in cognition (enough to be characterized as a mildly cognitively impairment) over the course of 78.75 years. In other words, it is expected that cognitively normal older individuals aged 50 years and older would need more than double their current age to exhibit aging-related decrements in performance equivalent to those observed in the $\mathrm{AD}$-pattern participants. 
Table 3. Coefficients and standard deviations examining associations between age, stroke, and Alzheimer's disease in explaining longitudinal declines in orientation

\begin{tabular}{|c|c|c|c|c|}
\hline \multirow[t]{2}{*}{ Fixed effect } & \multicolumn{2}{|l|}{ Model 1* } & \multicolumn{2}{|l|}{ Model 2* } \\
\hline & mean & $\mathrm{SD}$ & mean & SD \\
\hline Male sex & 0.450 & 0.028 & 0.403 & 0.027 \\
\hline Age, years & -0.064 & 0.001 & -0.035 & 0.001 \\
\hline \multicolumn{5}{|l|}{ Race/ethnicity } \\
\hline White & Reference & & Reference & \\
\hline Black & -2.141 & 0.040 & -2.082 & 0.040 \\
\hline Other & -1.213 & 0.096 & -1.141 & 0.094 \\
\hline Hispanic & -2.085 & 0.049 & -2.016 & 0.048 \\
\hline Stroke & & & -0.357 & 0.022 \\
\hline Alzheimer's disease & & & -0.384 & 0.005 \\
\hline Constant & 14.326 & 0.028 & 13.936 & 0.027 \\
\hline \multirow[t]{2}{*}{ Random effect } & \multicolumn{2}{|l|}{ Model 1* } & \multicolumn{2}{|l|}{ Model 2* } \\
\hline & ES & SE & ES & SE \\
\hline Slopes, SD & 0.114 & 0.001 & 0.096 & 0.001 \\
\hline Intercepts, SD & 1.544 & 0.013 & 1.462 & 0.013 \\
\hline $\operatorname{Corr}(\mathrm{S}, \mathrm{I})$ & -0.124 & 0.014 & 0.066 & 0.018 \\
\hline
\end{tabular}

SE, standard error; ES, estimate size; SD, standard deviation. * All nonzero associations shown were statistically significant at $p<1 \mathrm{E}-06$ level. In addition to covariates shown, all analyses additionally adjusted for practice effect observed between the first and following waves.

Analyses examining change in orientation provided similar overall results (Table 3), suggesting that episodic memory declines consistent with AD were characterized by the loss of 0.384 points/year in orientation, a decline that explained approximately $51.07 \%$ [40.70-46.06] of aging-related declines in orientation. This loss was larger than the association with stroke and equivalent to onepoint loss in orientation every 2.60 years or one SD loss in orientation in 6.46 years. Integration of $\mathrm{AD}$ and stroke variables accounted for the negative association between individual random intercepts and slopes and even predicted an inverted association such that improved orientation was slightly associated with slower rates of cognitive decline.

Sensitivity analyses were conducted integrating a quadratic estimate of acceleration in cognitive decline. Results from this study suggested that quadratic age was associated with acceleration in decline $(B=-0.0034)$ when included in models not adjusting for $\mathrm{AD}$ or stroke, but this association was reduced by $61.76 \%$ in size $(B=$ -0.0013 ) upon adjustment for Alzheimer's disease and stroke. In those models, both $\mathrm{AD}$ and stroke retained 96.88 and $97.49 \%$ of their effect size estimates, respectively. Additionally, estimates of model fit $\left(\sim R^{2}=0.132\right.$ when including $\mathrm{AD} /$ stroke and quadratic vs. 0.132 for models only including $\mathrm{AD} /$ stroke but 0.104 for models only incorporating quadratic forms) suggested that inclusion of the quadratic form was limited in impact. Finally, examinations of decline in the "mixed pathology" group were completed to characterize differences in this group. Results from analyses of this group suggested that declines attributed to both $\mathrm{AD}$ and stroke were highly significant $(p<1 \mathrm{E}-06)$ and that effect sizes were stronger in this group for $\mathrm{AD}(B=-0.80 /$ year $)$ and for stroke $(B=$ $-1.24)$ than those presented in the models, potentially suggesting that this group is experiencing more severe cognitive decline than other groups.

\section{Discussion}

Clinical diagnosis of cognitively impairing conditions such as Alzheimer's disease (AD) and stroke relies heavily on subjective reports to differentiate between potential root causes of precipitous cognitive decline. However, we propose that the earliest phases of vascular injury or AD can be detected using objective measurements in large longitudinal datasets. In this analysis, we utilized a pattern detection algorithm to account for $\mathrm{AD}$ and acute stroke events to differentiate patterns of clinical cognitive impairment from age-related cognitive decline evident in healthy older adults. We found that $\mathrm{AD}$ and stroke explained more than half of the association between age and verbal episodic memory, as well as the association between age and orientation. Separating out individuals who exhibited longitudinal patterns of performance indicative of $\mathrm{AD}$ and stroke removed variation commonly attributed to random slopes in longitudinal multilevel modeling. Additionally, adjusting for $\mathrm{AD}$ and stroke explained most of the variation previously attributed to random slope estimates in the multilevel model.

Improving the ability of clinicians to make diagnoses earlier in the disease course offers opportunities to lower patient risks and prevent complications, and may also help individuals better prepare for their future [21]. Thus, there is a need to develop methods to reliably identify patterns associated with clinical levels of cognitive decline and to accurately identify methods for reliably diagnosing the etiology of the impairment [22]. Years of preclinical cognitive decline in longitudinal data fits the natural history of $\mathrm{AD}$ and, in turn, implies the possibility of utilizing 
population-based data mining techniques to improve identification. Distinct from models relying on neuropsychological test cutoffs and using random slope methods, the current method reinterprets a long-standing debate emphasizing the critical importance of patterns of within-person change over time [23].

Results suggesting that AD and stroke can help explain the association between age and cognitive function are supportive of work in the neuropathological field showing similar results. For example, recent work relying on the Mayo Clinic Study of Aging utilized positron emission tomography to show that $52 \%$ of the association between age and cognitive decline could be explained by cerebrovascular changes, amyloid deposition, and neurodegeneration [4]. Similarly, results using histopathological factors suggest that $41 \%$ of cognitive decline could be attributed directly to neuropathology, including amyloid or Lewy bodies observed in the brain at autopsy [5]. However, less is known about the potential for mild cognitive dysfunction to emerge as a result of cerebrovascular disease, since a large amount of cerebrovascular disease is asymptomatic [24]. Further work is warranted to determine what types of cerebrovascular disease have cognitive profiles but lack symptoms of dementia.

The results of this study suggested that the rate of decline in episodic memory was similar in this group $(-0.064$ words or approximately -0.02 SDs in Table 3$)$ as compared to previous reports of declines identified at rates of -0.024 in episodic memory [25], although other cognitive domains ranged from -0.003 to -0.096 SDs in size. The current study noted that -0.024 might be an overestimate that was negatively biased due to the inclusion of a large number of participants with preclinical and undiagnosed $\mathrm{AD}$ and/or stroke. Future research is warranted to determine measures of cognition that are less sensitive to both stroke and $\mathrm{AD}$ neuropathology in order to better understand the rate of change attributable to aging alone.

Complementary evidence to the data presented here suggest that this might be a fruitful avenue to explore. For example, performance on an associative memory task has been shown to predict conversion rates from amnestic mild cognitive impairment to early-stage $\mathrm{AD}$ [26], but associative memory performance has been shown to be relatively spared in stroke patients [27] in comparison to age-matched healthy controls. Although extant data suggest that associative memory deficits are common even in healthy aging when compared to performance in younger cohorts [28], longitudinal assessment of performance over time in this domain may prove useful in differentiating cognitive profiles associated with healthy aging, $\mathrm{AD}$, and stroke. Moving beyond the domain of memory, executive dysfunction is often observed even in very early stages of AD [29] and may serve as an early warning sign for risk of conversion to AD [30]. Executive dysfunction is also commonly observed in acute stroke patients [31]. However, longitudinal change in executive dysfunction should be examined to assess whether trajectories of change in this domain differ meaningfully at later stages of disease progression.

The specific pathologies that the pattern recognition method is designed to detect, while useful, are not well understood [32]. This lack of clarity represents a huge challenge to understanding both individual clinical diagnoses and diagnostic prevalence at the population level because gold-standard diagnoses often rely on expensive neuroimaging or postmortem evidence [33]. Lacking evidence from neuroimaging, prior efforts relied on individuals or their spouses to determine timing of symptoms in order to make a differential diagnosis [34], and as a result, most cases of both $\mathrm{AD}$ [35] and stroke [36] may remain unobserved at death. Because these states are difficult to disentangle from processes that occur in the context of normal aging, studies rarely make a clear distinction between causes of functional decline [37]. This study is, therefore, unique in utilizing new methods to identify stroke and $\mathrm{AD}$ pathologies that have otherwise gone undetected, and in differentiating these trajectories from the rate of age-related cognitive decline observed in healthy older adult populations.

An increasing body of research has highlighted the importance of mixed $\mathrm{AD}$ and stroke as a crucial area of interest as many individuals lacking cerebrovascular disease do not progress in $\mathrm{AD}$ despite evidence of amyloidosis [38]. One explanation for this effect that has been offered is that microglia, which are activated in cerebrovascular disease [39], are also central to more rapid proliferation of protein-tau [40]. Additionally, researchers have posited that the amyloid cascade may require both the deposition of $\beta$-amyloid 1-42 throughout the cerebrum and the inclusion of $\beta$-amyloid 1-40 in the vasculature in order to cause neurodegeneration [41]. In either case, these results support a growing body of research suggesting that $\mathrm{AD}$ is worsened by the presence of cerebrovascular disease. Further research is warranted in this group to determine risk and protective factors and also to identify indicators of cognitive reserve and brain resilience in this group. 


\section{Limitations}

This study is one of the first to specifically focus on modeling both healthy aging and different causes of cognitive decline using pattern recognition. Nevertheless, this method is designed to identify individuals with possible stroke and/or $\mathrm{AD}$, rather than provide definitive diagnoses. Although this study has identified a potentially large cerebrovascular burden in older age, further work is needed to validate both patterns of cognitive decline in Alzheimer's disease and stroke with biomarker data.

Although episodic memory is recognized as a central symptom for neuropathological injury, and to measure brain aging, strokes not fitting the pattern utilized for classification may be missed. Missing data were not used to inform pattern detection routines as they were deemed to be beyond the scope of this study. Respondents in the current study included a wide array of American residents from disparate social and racial backgrounds. Nevertheless, further work should examine racial/ethnic subgroups to determine whether the efficacy of stroke detection efforts is modified by demographic characteristics. The current study was limited to including only individuals with a large number of follow-ups occurring over a relatively long period of time, biasing these results to favor individuals who are healthier since they had to continue responding after the onset of $\mathrm{AD}$ symptoms and, more importantly, stroke. Additionally, although individuals with the patterns identified are likely to fit the diagnostic criteria for AD and stroke-related cognitive decline, we did not incorporate information about the presence of neuropathological indicators to positively determine differential diagnosis.

Previous research has pointed to the possibility that stroke can produce subtle changes that may make diagnosis difficult [24], and therefore, we may have missed cases of stroke that presented with subtle or atypical symptoms. Additionally, our algorithm was only designed to identify a single stroke event, thereby making it possible that individuals with multiple overlapping strokes were misclassified, with the most likely end result being inappropriate attribution as rapid aging or as $\mathrm{AD}$. Future work is warranted to increase sensitivity of the algorithm to identifying multiple strokes over time and also to identifying other cognitively impairing diseases such as dementia due to Lewy bodies. Bias due to misspecification of the period of onset was not quantified in this study; future work is warranted using simulation to examine the extent to which misspecification of timing influences model results. Finally, we only reported linear models for normal cognitive aging, in part because quadratic models were not highly informative after adjusting for $\mathrm{AD}$ and cerebrovascular disease burden.

\section{Implications}

Results from this study support prior work suggesting that approximately half of age-related decline in episodic memory and orientation in old age may be attributable directly to relatively common diseases including stroke and $\mathrm{AD}$, and even suggest that the modal experience of cognitive change in old age is neuropathologic. Episodic memory is important for maintaining quality of life, and being able to mentally replay past events is a key component of good episodic memory and serves to reinforce continuity and sense of self [42], improve quality of life and mental health [43], and reduce the impact of caregiver burden [44]. However, as more older adults struggle with cognitive decline in old age, these analyses support the increasing view that neuropathologic changes may be responsible for a large proportion of that decline.

\section{Acknowledgements}

The Health and Retirement Study is sponsored by the National Institute on Aging (grant number NIA U01AG009740) and is conducted by the University of Michigan. The authors would like to thank the National Institute on Aging for supporting this work (NIH/NIA R01 AG058595).

\section{Statement of Ethics}

This study was determined by the Stony Brook IRB to be not human subjects research.

\section{Conflict of Interest Statement}

The authors have no conflicts of interest to disclose.
1 Alzheimer's Association. 2016 Alzheimer's disease facts and figures. Alzheimers Dement. 2016;12(4):459-509.

2 Alzheimer's Association. 2015 Alzheimer's disease facts and figures. Alzheimers Dement. 2018;11(3):332-84.

3 Gorelick PB, Scuteri A, Black SE, DeCarli C, Greenberg SM, Iadecola C, et al. Vascular contributions to cognitive impairment and dementia: a statement for healthcare professionals from the American Heart Association/American Stroke Association. Stroke. 2011 Sept 1;42(9):2672-713 
4 Vemuri P, Lesnick TG, Knopman DS, Przybelski SA, Reid RI, Mielke MM, et al. Amyloid, vascular, and resilience pathways associated with cognitive aging. Ann Neurol. 2019; 86(6):866-77.

5 Boyle PA, Wilson RS, Yu L, Barr AM, Honer WG, Schneider JA, et al. Much of late life cognitive decline is not due to common neurodegenerative pathologies. Ann Neurol. 2013; 74(3):478-89.

6 Gustafson L, Nilsson L. Differential diagnosis of presenile dementia on clinical grounds. Acta Psychiatr Scand. 1982;65(3):194-209.

7 McKhann GM, Knopman DS, Chertkow H, Hyman BT, Jack CR, Kawas CH, et al. The diagnosis of dementia due to Alzheimer's disease: recommendations from the National Institute on Aging-Alzheimer's Association workgroups on diagnostic guidelines for $\mathrm{Al}$ zheimer's disease. Alzheimers Dement. 2011; 7(3):263-9.

8 Salthouse TA. Are individual differences in rates of aging greater at older ages? Neurobiol Aging. 2012 Oct;33(10):2373-81.

9 Bruscoli M, Lovestone S. Is MCI really just early dementia? A systematic review of conversion studies. Int Psychogeriatr. 2004;16(2): $129-40$.

10 Richards M, Deary IJ. A life course approach to cognitive capability. In: Kuh D, Cooper R, Hardy R, Richards M, Ben-Shlomo Y, editors. A life course approach to healthy ageing. Oxford: Oxford University Press; 2014. p. 32-45.

11 Schneider BC, Gross AL, Bangen KJ, Skinner JC, Benitez A, Glymour MM, et al. Association of vascular risk factors with cognition in a multiethnic sample. J Gerontol B Psychol Sci Soc Sci. 2014;70(4):532-44.

12 Clouston SA, Glymour M, Terrera GM. Educational inequalities in aging-related declines in fluid cognition and the onset of cognitive pathology. Alzheimers Dement. 2015 Sep 1; 1(3):303-10.

13 Clouston SAP, Zhang Y, Smith DM. Pattern recognition to identify stroke in the cognitive profile: secondary analyses of a prospective cohort study. Cerebrovasc Dis Extra. 2019; 9(3):114-22.

14 Health and Retirement Study (HRS). Health and retirement study, public use dataset. In: University of Michigan with funding from the National Institute on Aging (U01 AG 009740), editor. Ann Arbor: HRS; 2014.

15 Bäckman L, Small BJ, Fratiglioni L. Stability of the preclinical episodic memory deficit in Alzheimer's disease. Brain. 2001 Jan;124(Pt 1):96-102.

16 Dregan A, Wolfe CD, Gulliford MC. Does the influence of stroke on dementia vary by different levels of prestroke cognitive functioning? A cohort study. Stroke. 2013;44(12): 3445-51.

17 Clouston SAP, Zhang Y, Smith DM. Pattern recognition to identify stroke in the cognitive profile: secondary analyses of a prospective cohort study. Cerebrovasc Dis Extra. 2019; 9(3):114-22.
18 Sliwinski M, Buschke H. Modeling intraindividual cognitive change in aging adults: results from the Einstein aging studies. Aging Neuropsychol Cogn. 2004;11(2-3):196-211.

19 Rabe-Hesketh S, Skrondal A. Multilevel and longitudinal modeling using Stata. College Station: STATA Press; 2008.

20 Liu GF, Lu K, Mogg R, Mallick M, Mehrotra DV. Should baseline be a covariate or dependent variable in analyses of change from baseline in clinical trials? Stat Med. 2009;28(20): 2509-30.

21 Doubal FN, Ali M, Batty GD, Charidimou A, Eriksdotter M, Hofmann-Apitius M, et al. Big data and data repurposing: using existing data to answer new questions in vascular dementia research. BMC Neurol. 2017 April 17;17(1):72.

22 Albert MS, DeKosky ST, Dickson D, Dubois B, Feldman HH, Fox NC, et al. The diagnosis of mild cognitive impairment due to $\mathrm{Al}$ zheimer's disease: recommendations from the National Institute on Aging-Alzheimer's Association workgroups on diagnostic guidelines for Alzheimer's disease. Alzheimers Dement. 2011 May;7(3):270-9.

23 Berg L, Hughes CP, Coben LA, Danziger WL, Martin RL, Knesevich J. Mild senile dementia of Alzheimer type: research diagnostic criteria, recruitment, and description of a study population. J Neurol Neurosurg Psychiatry. 1982;45(11):962-8

24 Yousufuddin M, Young N. Aging and ischemic stroke. Aging. 2019;11(9):2542-4.

25 Ritchie SJ, Tucker-Drob EM, Cox SR, Corley J, Dykiert D, Redmond P, et al. Predictors of ageing-related decline across multiple cognitive functions. Intelligence. 2016;59:115-26.

26 Irish M, Lawlor BA, Coen RF, O'Mara SM. Everyday episodic memory in amnestic mild cognitive impairment: a preliminary investigation. BMC Neurosci. 2011;12(1):80

27 van Geldorp B, Kessels RP, Hendriks MP. Single-item and associative working memory in stroke patients. Behav Neurol. 2013;26(3): 199-201.

28 Old SR, Naveh-Benjamin M. Differential effects of age on item and associative measures of memory: a meta-analysis. Psychol Aging. 2008;23(1):104-18.

29 Lafleche G, Albert MS. Executive function deficits in mild Alzheimer's disease. Neuropsychology. 1995;9(3):313, 20.

30 Carlson MC, Xue QL, Zhou J, Fried LP. Executive decline and dysfunction precedes declines in memory: the women's health and aging study II. J Gerontol A Biol Sci Med Sci. 2009;64(1):110-7.

31 Zinn S, Bosworth HB, Hoenig HM, Swartzwelder HS. Executive function deficits in acute stroke. Arch Phys Med Rehabil. 2007; 88(2):173-80.

32 Vitali P, Migliaccio R, Agosta F, Rosen HJ, Geschwind MD. Neuroimaging in dementia. Semin Neurol. 2008 10/08;28(4):467-83.
33 Scheltens P, Blennow K, Breteler MM, de Strooper B, Frisoni GB, Salloway S, et al. Alzheimer's disease. Lancet. 2016 Jul 30; 388(10043):505-17.

34 Pohjasvaara T, Mäntylä R, Ylikoski R, Kaste $\mathrm{M}$, Erkinjuntti T. Comparison of different clinical criteria (DSM-III, ADDTC, ICD-10, NINDS-AIREN, DSM-IV) for the diagnosis of vascular dementia. National Institute of Neurological Disorders and Stroke-Association Internationale pour la Recherche et l'Enseignement en Neurosciences. Stroke. 2000 Dec;31(12):2952-7.

35 Boustani M, Peterson B, Hanson L, Harris R, Lohr KN. Screening for dementia in primary care: a summary of the evidence for the U.S. Preventive Services Task Force. Ann Intern Med. 2003;138(11):927-37.

36 Ince PG, Minett T, Forster G, Brayne C, Wharton SB, Function MRCC, et al. Microinfarcts in an older population-representative brain donor cohort (MRC CFAS): prevalence, relation to dementia and mobility, and implications for the evaluation of cerebral small vessel disease. Neuropathol Appl Neurobiol. 2017;43(5):409-18

37 Korczyn AD, Vakhapova V, Grinberg LT. Vascular dementia. J Neurol Sci. 2012 05/08; 322(1-2):2-10.

38 Mielke MM, Rosenberg PB, Tschanz J, Cook L, Corcoran C, Hayden KM, et al. Vascular factors predict rate of progression in $\mathrm{Al}$ zheimer disease. Neurology. 2007;69(19): 1850-8.

39 Hu X, Li P, Guo Y, Wang H, Leak RK, Chen $S$, et al. Microglia/macrophage polarization dynamics reveal novel mechanism of injury expansion after focal cerebral ischemia. Stroke. 2012;43(11):3063-70.

40 Pampuscenko K, Morkuniene R, Sneideris T, Smirnovas V, Budvytyte R, Valincius G, et al. Extracellular tau induces microglial phagocytosis of living neurons in cell cultures. J Neurochem. 2019:e14940.

41 Kritikos M, Clouston SAP, Diminich ED, Deri Y, Yang X, Carr M, et al. Pathway analysis for plasma $\beta$-amyloid, tau and neurofilament light (ATN) in world trade center responders at midlife. Neurol Ther. 2020;9(1): $159-71$.

42 Conway MA. Sensory-perceptual episodic memory and its context: autobiographical memory. Philos Trans R Soc Lond, B, Biol Sci. 2001;356(1413):1375-84.

43 Clouston SAP, Diminich ED, Kotov R, Pietrzak RH, Richards M, Spiro A 3rd, et al. Incidence of mild cognitive impairment in World Trade Center responders: Long-term consequences of re-experiencing the events on 9/11/2001. Alzheimers Dement. 2019 Dec; 11:628-36.

44 Elif K, Taşkapilioğlu Ö, Bakar M. Caregiver burden in different stages of Alzheimer's disease. Arch Neuropsychiatry. 2017;54(1):82.
Pattern Recognition to Differentiate Etiology of Cognitive Decline
Neuroepidemiology 2020;54:446-453

DOI: $10.1159 / 000510133$ 